Monatsschrift f. Geburtshülfe u. Gynäkologie 1931;88:413-422

\title{
Gedenkworte an Otto Küstner †
}

\section{Von}

\section{WALTHER HANNES.1)}

Meine Damen und Herren! Wenn ich Ihnen hier die Persönlichkeit Otto Küsíners - mit Fug und Recht kann ich wohl sagen, unseres Otto Küstner - eindrucksvoU und lebendig vor Augen führen soil, so ist dies, so widerspruchsvoll es

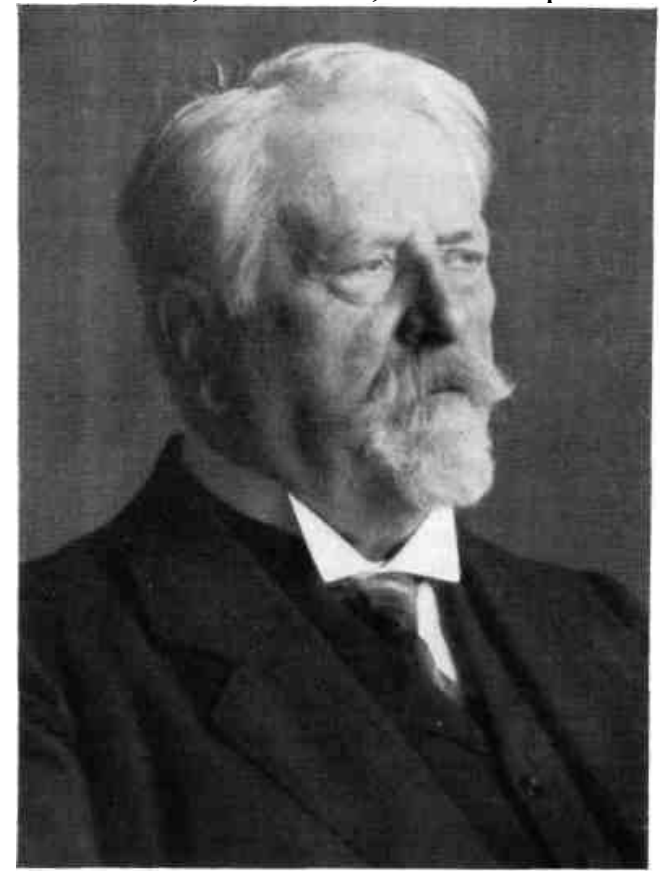

auch scheinen mag, sowohl eine leichte und schöne und mir besonders Hebe Aufgabe und andererseits eine vielgestaltige und infolgedessen unendlich schwierige, ja mir kaum erfüllbar erscheinende Aufgabe. Kaum erfüllbar deswegen, weil ich mich nicht für beredt genug halte, um alles und jedes seines Wesens und seiner Art mit genügender Schärfe und Lebenswärme darzulegen und mit genügender Lebendigkeit Aus-druck zu verleihen; schön, leicht und besonders lieb mir aber dadurch,

a) Gesprochen am 11. Juni 1931 in der Gynäkologischen Gesellschaft zu Breslau. 414

Hannes, Gedenkworte an Otto Küstner.

daß es mir als dem Schüler, dem es vergönnt war, mehr als 20 Jahre des inhaltvollen, arbeitsund erfolgreichen Lebens Otto Küsíners in seiner nächsten Umgebung mitzugehen, mitzuerleben und lernend mit-zuarbeiten - daß es nun gerade mir obliegt, hier in Ihrem Kreise zu reden, in welchem ja noch die meisten Otto Küstner persönlich kannten und ihm kerneswegs fernstanden. Ja ich glaube, ohne Übertreibung sagen und behaupten zu können, daß alle diejenigen Mitglieder unserer Gesellschaft, die Otto Küstner noch als aktives Mitglied erleben durften, sich als seine 
indirekten Schüler selbst dann betrachten, wenn sie bei ihm weder Student, noch Praktikant oder Assistent gewesen waren.

Nachdem Otto Küstner im Jahre 1902 gemeinsam mit unserem jetzigen Ehrenmitglied Fríedrích Weinhold diese gynäkologische Gesellschaft gegründet hatte, verstand er es, nicht nur sämtliche Breslauer Fachgenossen in diesem Kreis zu vereinigen, sondern auch die über-wiegende Mehrheit der schlesischen Frauenärzte außerhalb imserer Stadt zum Anschluß zu gewinnen. Man kann und muß es behaupten, daß die Kraft, die Otto Küstner in den Dienst unserer Gesellschaft stellte, das gedeihliche und reibungslose Arbeiten unserer Gesellschaft, ihr Aufblühen im ersten Vierteljahrhundert ihres Bestehens, die weit-gehende Anerkennung und Achtung, die sie sich im Kreis ihrer Schwestergesellschaften erwerben konnte, bewirkte. Bis zu seinem Weg-zug von Breslau im Frühjahr 1923, also während mehr als 20 Jahren, hat er nur sechsmal an unseren Sitzungen nicht teilnehmen können. Of fen und dabei nie verletzend oder taktlos, nahm er stets und zu alien hier in unserem Kreis diskutierten Fragen Stellung. Und eben gerade seine Ausführungen in der Diskussion hat ihm die Mitglieder unserer Gesellschaft nähergebracht; so wurden wir alle seine Schüler, alle lernten wir aus seinem Vorbild, seiner Erfahrung, aus dem reichen Schatz seines Wissens und Könnens, das er uns hier rückhaltlos, jedoch ohne Anspruch auf unbedingte oder gar dogmatische Anerkennung voll und ganz offenbarte. Wieviele seiner Forschungsergebnisse und epochalen Arbeiten Otto Küstner in diesem Kreis zuerst bekannt gab, brauche ich ja hier nicht weiter auszuführen. Unsere Gesellschaft war gewissermaßen eines seiner jüngsten Kinder, denen man ja doch eben oft besonders zugetan ist.

So war es ja auch uns vergönnt, das letzte schöne Fest seines Lebens, seinen 80. Geburtstag, mit ihm hier in Breslau noch besonders feiern zu dürfen. Und gerade im Oktober 1929 war unser damaliger Jubilar von so lebensvoller, lebensfrischer Energie begabt; Otto Küstners lebensbejahendes Temperament sprudelte damals noch so völlig ungebrochen, ja begeisternd und jugendlich begeistert, daß wohl jeder von uns diesem körperlichen und geistigen reckenhaften Hünen noch recht viele ungetrübte Lebens jahre nicht nur herzlichst wünschte, sondern sie ihm auch als sicheren Besitz eigentümlich meinte. Und doch - niemand in diesem Kreise hätte dies vermutet oder geahnt - sollte es unser letztes Beisammensein mit unserem großen Meister sein. Genugsam hat wohl nach alledem gerade unsere Gesellschaft An-laß, unseren teueren Entschlafenen, ihren Gründer und Ehrenvorsitzen-den, durch diese feierliche Gedenkstunde zu ehren, seiner dabei einHannes, Gedenkworte an Otto Küstner.

415

gehend zu gedenken und das Bild dieses großen Mannes, das Sein und Schaffen Otto Küstners sich vor Augen zu führen.

Wenn ich dies jetzt vor Ihnen, meine Damen und Herren, zu tun versuche, bin ich mir wohl bewußt, daß dies im Rahmen solch einer Rede gar nicht möglich ist, daß jedem von Ihnen so manches und vor allem so manches Bedeutsame aus Otto Küstners Leben und Schaffen, aus seinem Lehren und Wirken bekannt sein dürfte, was hier aus Zeit-mangel nicht zur Sprache kommen kann, was aber noch viel zur leb-haften Fixierung seiner Persönlichkeit in unserem geistigen Auge für alle Zeiten beitragen könnte.

Ganz kurz, meine Damen und Herren, einige wenige biographische Daten aus seinem Leben: Geboren am 26. August 1849 auf dem väterlichen Rittergut Trossin, absolvierte er das Gymnasium in Torgau, studierte dann in Leipzig, Berlin und Halle; sein Studium wurde unterbrochen durch den Krieg 1870/71, den Küstner als Student im fünften Semester mit der Waffe mitmachte. Nach beendetem Staatsexamen war er Assistent beim Internisten Th. Weber in Halle 
und dann ebenda bei Olshausen; nachdem Küstner sich in Halle 1877 habilitiert hatte, siedelte er bald als Assistent und Dozent zu B. S. Schultze nach Jena über, wo er 1879 a. o. Professor wurde. Seit Ende 1887 Ordinarius in Dorpat, folgte Küstner im Herbst 1893 dem Ruf nach Breslau, wo er nahezu 30 Jahre, bis zu seiner am 28. Februar 1923 erfolgten Emeri-tierung, lebte, lehrte und wirkte.

Hier in Breslau ist Otto Küstner dann bodenständig geblieben, trotz mancher Rufe, die aus anderen Universitäten an ihn ergingen. Die Breslauer geburtshilflich-gynäkologische Schule wurde zur Küstner-schen Schule.

Diese drei Jahrzehnte hier in Breslau sind zum Kernpunkt Otto Küstners Lebens und Wirkens geworden. Nicht leicht war es, als Nach-folger Heinrich Frítschs sich Ansehen, Wertschätzung, Liebe und Ver-ehrung zu schaffen. Ja manche Kreise der Breslauer und Schlesischen Arzteschaft, ja auch die klinischen Studenten standen dem neuen, aus dem zaristischen Rußland kommenden Professor zum mindesten recht skeptisch gegenüber. Und hier in Breslau neben den Koryphäen der ärztlichen Wissenschaft und der Fakultät - ich nenne nur Heiden-hain, Ponfíck, Flügge, Mikulicz, Kast, Neisser - wurde Otto Küstner nicht nur selbst bald ein Stolz der Fakultät, er wurde auch zu einem der beliebtesten klinischen Lehrer. So konnte sich auch die Breslauer Universität, als am 1. August 1914 die Not des Weltkriegs unabwendbar war, keinen besseren und geeigneteren Rektor für das erste Kriegsjahr, das ja gerade für Breslau so viele Gefahren brachte, erwählen als Otto Küstner. Als er dann im 74. Lebensjahr - ein immer noch besonders temperamentvoller klinischer Lehrer - von seinem Lehramt schied, waren Generationen deutscher Ärzte froh und stolz, sich als seine Schüler bezeichnen zu dürfen. Ein besonderer Genuß war sein klinischer Unterricht. So mannig-fach auch - ich habe selbst wohl zirka 30 Semester die Klinik regel-mäßig mitgehört - das in jeder klinischen Stunde den Studenten ge-botene Material an Untersuchungsfällen, Geburtsberichten, makroMonatsschrift f. Geburtshülfe u. Gynäkologie. Bd. LXXXVПI. Heft 5.27 416 Hannes, $\mathrm{G}^{1} \frac{1}{8}$ denkworte an Otto Küstner. skopischen und mikroskopischen Präparaten war, so war es doch ge-radezu genial, ungemein belebend und belehrend, wie Otto Küstner es verstand, in jeder klinischen Stunde in Anlehnung an das vorgeführte Material über ein bestimmtes Thema, über einen Ausschnitt aus dem Wissens- und Forschungsgebiet unseres Fachs einen in sich ge-schlossenen, wissenschaftlich und praktisch erschöpfenden, ja geradezu druckfertigen klinischen Vortrag zu halten.

Gesehwänzt wurde bei Otto Küstner kaum je, dafür aber um so mehr von jungen klinischen Semestern geschunden. Jetzt eben auf dem Frankfurter Kongreß der Deutschen Gesellschaft für Gynäkologie sagte mir der Ordinarius eines theoretisch-medizinischen Fachs an einer ausländischen Universität, der in Breslau klinischer Student gewesen war, wie oft und mit welcher Freude und Begeisterung er jetzt immer noch gerade an Küstners klinischen Unterricht denke.

Temperamentvoll und begeistert für sein Lehramt, scheute Otto Küstner im Unterricht und im Examen auch nicht davor zurück, einmal of fen und sanguinisch ein wenig derb zu kritisieren. Mancherlei bons mots waren und sind ja darüber im Umlauf. Eine lebhafte und zum Teil ungemein witzige Sprache redeten davon und darüber die Wand-bemalungen im Praktikantenkasino und die Kliniker-Bierzeitungen. Ihn selbst hat das immer besonders gefreut die Wandbemalungen waren bei jeder Renovation des Praktikantenkasinos tabu - und ihm selbst hat seine gelegentliche offene Kritik bei den Betroffenen nie Feinde, sondern nur Freunde geschaffen. Schnitt bei ihm in der Prüfung ein Kandidat schlecht ab, dann ventilierte er mit uns 
stets eingehend die Frage, ob und wie dies vom Dozenten wohl verschuldet und von diesem aus zu bessern sei. So kritisch er gegen sich selbst war, so nachsichtig war er gegen andere. Wenn er z. B. in seiner Eigenschaft als gerichtlicher Gutachter über Mißerfolge, über sogenannte Kunstfehler anderer Ärzte urteilen sollte, so war sein oberstes Gesetz, aus dem seine Nachsicht ent-sprang, daß der, welcher bei einem solchen Unglücksfall nicht mit dabei gewesen sei, kaum objektiv urteilen und vor allem nicht verurteilen könne.

Immer wußten die Studenten, daß sie an ihm eine Stütze und Halt, Verständnis und Hilfe für ihre Fragen und Sorgen fanden. Ein Vorfall - nach meiner Kenntnis einzigartig und doch so bezeichnend für Otto Küstners Art - ist mir im Gedächtnis, dem ich selbst bei-wohnte, und den ich Ihnen nicht vorenthalten möchte. Küstner war Dekan und in dieser Eigenschaft damals Vorsitzender bei der Physikums-prüfung. Ein recht armer, gescheiter und fleißiger Student, den Otto Küstner persönlich gar nicht weiter kannte, war zum Erstaunen aller Anwesenden beim Physikexaminator ciurchgefallen. Er wurde sogleich beim Dekan vorstellig, und Otto Küstner bewog zu aller Erstaunen und Freude den Examinator, 0. E. Meyer, den Pruning sogleich noch einmal zu prüfen, was nunmehr zu einem Bestehen der Prüfung führte.

Die am Ende seiner Dorpater Zeit erstmalig erschienenen Grund-züge der Gynäkologie gestaltete Otto Küstner hier in Breslau, unter Heranziehung namhafter Fachgenossen, zu seinem , Kurzen Lehrbuch der Gynäkologie" um, welches von .1901 bis 1922 in neun Auflagen

Hannes, Gedenkworte an Otto Küstner.

417

erschien und während mehr als 20 Jahren das führende gynäkologische Lehrbuch nicht nur im deutschen Sprachgebiet war, und dessen Rolle als solches m. E. noch keineswegs ausgespielt ist. Hier in Breslait stellte Otto Küstner seine Schule auf eine viel breitere Basis, als dies in Dorpat im Kreis der ihm allerdings besonders liebgewordenen Schüler, von denen ich nur von Knorre, Weídenbaum, Holowkow, A. Keümann nennen will, möglich gewesenwar. Nichtwenige Russen und vor allem Balten kamen bis zum Weltkrieg auch noch nach Breslau, um bei Küstner zu hören und bei ihm Assistent zu werden. - Da es mir vergönnt war, während mehr als 20 Jahren von 1901 bis 1923 als Assistenzarzt, Oberarzt und zuletzt als Leiter der gynäkologischen Poliklinik dem engeren und engsten Schülerkreis Otto Küstners anzu-gehören, so seien mir auch hierüber einige Worte verstattet.

Otto Küstner war ein vorbildlicher Chef. Er verlangte von semen Assistenten dieselbe Pflichterfüllung wie von sich selbst; er machte uns zu seinen Schülern nicht mit Zwang, sondern durch sein Vorbild. Jeg-liche Pedanterie, jegliche Bürokratie lag ihm, dem wahrhaft großen Mann, vollkommen fern. Ungemein anregend und stets zum Eingehen auf wissenschaftliche Fragen und Plane bereit, lehnte er es strikt ab, einem jungen Volontär oder Assistenzarzt, der darum bat, ein Thema zu einer wissenschaftlichen Arbeit zu stellen. Die Absicht, sich mit einer wissenschaftlichen Fragestellung zu beschäftigen, einem Problem unseres Fachs mit eigenen Untersuchungen nachzugehen, sollte - so wollte es der Chef - immer in uns selbst reifen. Kam man dann mit einer Eragestellung, ob dies oder jenes bearbeitungsbedürftig sei, dann fand man bei ihm weitgehendste, freudige und fördernde Unterstützung. Otto Küstner war ein besonders glänzender Kenner unserer Eachliteratur. Kam man mit einer Erage, einer Problemstellung zu ihm, dann war ihm sofort gegenwärtig, was und vom wem etwas Wesentliches zu dem betreffenden Thema gearbeitet worden war. Nicht nur sein Lehrbuch, sondern vor allem auch seine Handbuchbeiträge im P. Müller, Veit, Doederlein, im Payr-Zweifel sind gewichtige Dokumente wirklich um-fassender, erschöpfender Literaturkenntnis und kritischster Würdigung derselben auf Grund großer, eigener, selbständiger und unbeeinflußt selbst gedeuteter Erfahrung. 
Otto Küstner war kein den Betrieb und die klinische Assistenten-arbeit hemmender Vorgesetzter. Er war fur den älteren Assistenten nie der Chef, sondern der Berater, der Konsiliarius, zu dem man gern und oft ging. Jederzeit, bei Tag und bei Nacht, war er für seine klini-schen Patienten zu haben, wenn man ihn brauchte und holte. Es gab für ihn hier keinen Unterschied des Standes oder des Geldbeutels. Lag eine besonders schwerkranke Patientin auf der Klinik, ging es einer Operierten nicht nach Wunsch, dann kam er selbst oft viele Male täglich zu ihr, ganz gleichgültig, ob es eine einfache Arbeiterfrau oder eine Millionärin war. Selbst nur auf kurze Zeit bemessene projektierte Er-holungsreisen verschob er solcher armer Kranken wegen und verkürzte so seine Erholungszeit noch mehr. Er war durch und durch nicht nur Forscher und Wissenschaftler, sondern vor allem auch ein vorbildlich humaner Arzt.

$27^{*}$

418

Hannes, G-ede $\pi$ kworte an Otto Küstner.

War sein reiches, frühzeitig am Morgen beginnendes Tagewerk - wenn früh nicht Klinik oder Operation war, dann saß der Chef schon vor acht Uhr an seinem Arbeitstisch - getan nnd hatte er dann mit seiner wundervollen, leider viel zu früh einem tückischen Leiden erlegenen Frau den ,,Scheitniger" Spaziergang gemacht, dann konnte man Otto Küstner noch bis spat abends schaffend an seinem Schreib-tisch sehen. Das große Erkerfenster seines im Erdgeschoß gelegenen Arbeitszimmers war geradezu das historische Fenster der Maxstraße. War es abends erleuchtet, so konnte man den Chef an seinem großen Schreibtisch sitzen sehen, ihm gegenüber, solange sie lebte, seine nicht nur von ihm, sondern auch von uns, seinen nächsten Schülern, unvergessene Frau, ebenfalls mit einer Arbeit beschäftigt. Im Sommer saß Otto Küstner, solange es das Tageslicht zuließ, direkt in jenem historischen Erkerfenster an einem kleinen Tisch arbeitend, mit dem Gesicht zur Straße. Und wenn man, während er mitten in der Arbeit war, zu ihm kam, immer hatte er Zeit, um eingehend das, was man auf dem Herzen hatte, durchzusprechen und zu erörtern.

War dies academicus oder Sonntag, dann kam der Chef meist so gegen elf Uhr auf die Klinik und ging dann zum Oberarzt oder zu einem der älteren Assistenten aufs Zimmer, besprach zuerst mit ihm, was er für den klinischen Betrieb auf dem Herzen hatte; dann aber gab es stets anschließend eine richtig freundschaftlich gemütliche Aussprache über momentan die Allgemeinheit oder die Betreffenden selbst besonders interessierende Dinge und recht oft auch über wissenschaftliche Fragen, was für uns dann immer besonders anregend war.

Otto Küstner hatte für alle unsere großen, kleinen und kleinsten Sorgen Sinn, Herz und Verstand; alles durfte und konnte man mit ihm ohne Scheu, Rat heischend, durchsprechen; nichts Menschliches war ihm fremd. Stets suchte er dann zu raten und zu helfen. Es mag manchem verwunderlich erscheinen, daß ich dies hier heute mit vor-trage; doch tue ich dies mit guter Absicht und mit gutem Grund. Manchem, der nicht Gelegenheit hatte, mit ihm in nähere Fühlung zu kommen, der Otto Küstner nicht näher trat, erschien er wenig zu-gänglich, ja vielleicht unnahbar. Ja mitunter konnte er im ersten Augenblick etwas unwirsch ablehnen. Dies entsprang, wie er mir selbst einmal auseinandersetzte, einer gewissen, ihm eigentümlichen Befangen-heit. Ein so ausgezeichneter Redner und klinischer Lehrer er war, so war er nach seinem eigenen Ausspruch stets vor Beginn der Vor-lesung, einer Rede usw. befangen, und wie dies eben menschliche Eigen-tümlichkeit ist, hinter der scheinbaren Unnahbarkeit, hinter der schein-baren Rauheit verbarg sich die Befangenheit.

Mir selbst ist Otto Küstner immer körperlich und geistig als das Urbild eines allseitig lebenspendenden, unfällbaren Baumes erschienen; körperlich und geistig rastlos und voll 
Begeisterung arbeitend vom frühen Morgen bis zum späten Abend, gönnte er sich selbst wenig Erholung und Ferien, die er dann zum nicht kleinen Teil im Kreis seiner Familie auf seinem väterlichen Rittergut Trossin, das er ja auch zu seinem Alterssitz wählte, verlebte. Otto Küstner war seiner ganzen Anlage, seiner ganzen Entwicklung nach von Jugend auf eine ausgesprochen Hannes, Gedenkworte an Otto Küstner. 419

naturgebundene Persönlichkeit. Dem entsprach auch seine große Liebe und Freude an der Jagd, die ihm auch während der angestrengten Arbeitszeit gelegentlich einmal Veranlassung zum Ausspannen für einen oder wenige Tage gab, und der er bis fast in die letzte Zeit seines Lebens noch gelegentlich immer mit großer Freude nachging. Dabei war ihm der Aufenthalt und die Bewegung in Feld und Wald und die für den Jäger notwendige Naturbeobachtung die größte Freude.

Die ganze Entwicklung, die unser Fach von der Geburtshilfe über die Gynäkologie zur modernen Frauenkunde in den letzten 60 Jahren gegangen ist, hat Otto Küstner nicht nur miterlebt, sondern weitgehend mitgeschaf fen; sein Leben und sein Wirken ist zu einem Grundpfeiler unserer Disziplin geworden und dies auch für die Arbeitsgebiete und Betrachtungsformen der modernen Frauenkunde. Ich will hier nur an seine völlig modernen Anschauungen erinnern über Dinge, die jetzt modern als Konstitution zusammengefaßt werden, und deren Be-deutung für die Entstehung von Lageveränderungen und Prolaps, wie sie von Otto Küstner bereits vor Jahrzehnten entwickelt wurden; ähnlich liegen diese Dinge bei der modernen Wochenbettdiätetik. Wenn uns ja auch alien Otto Küstners überragende wissenschaft-liche Bedeutung klar und deutlich vor Augen steht, so muß ich als Schüler doch auch noch ein wenig auf dieses Gebiet eingehen, denn der Schüler, der so manche wissenschaftliche Großtat seines Lehrers hat reifen sehen, sieht hier manches doch mit anderen Augen. Wie eben nur ganz wenige überragende Geister hatte Otto Küstner die Genialität, immer gleichsam intuitiv das Wesentliche zu erfassen. Hatte er dann auf Grund exakter, kritischster, anatomischer, klinischer, biologischer Beobachtungen und Untersuchungen sich seine Meinung über ein Problem gebildet oder eine neue Operationsmethode ersonnen und durchgeführt, dann übergab er sie der wissenschaftlichen Offentlichkeit, scheute niemandes Kritik, sondern parierte sie sicher und treffend.

Nichtsdestoweniger war Otto Küstner von einer ganz besonderen wissenschaftlichen und publizistischen Bescheidenheit. Oft habe ich mit ihm über diesen Punkt gesprochen; nie konnte er sich bereit finden, wie das von so vielen geübt wird, eine Arbeit in verschiedener Aufmachung mehrfach zu veröffentlichen. Wenn z. B. Otto Küstner die publizistische Rührtrommel geschlagen hätte, so wüßte mindestens die breite ärztliche Offentlichkeit, vielleicht sogar die Laienwelt, daß die moderne Wochenbettdiätetik des Frühaufstehens sich eigentlich an den Namen Otto Küstner knüpft, und ganz ähnlich ist es mit der Me-thodik der direkten inneren Beckenmessung.

Grundlegenden Anted haben seine Arbeiten vor allem auch an dem Aufbau und Ausbau der operativen Seite unseres Fachs; manches, was Otto Küstner da vor Jahrzehnten als richtig erkannte und empfahl, ist dann in jüngster Zeit wenig verändert von anderer Seite wieder eindringlich empfohlen worden; hier möchte ich nur nennen seine Ansichten über die Bedeutung einmal des Morzellements für die Myomotomie und ferner der Ausgestaltung der Radikaloperation des Vulkakarzinoms. Otto Küstner war es, der die prinzipielle Bedeutung der Probepunktion des Douglas für die Differentialdiagnose gewisser gynäkologischer 420

Hannes, Gcdenkworte an Otto Küstner. 
Affektionen erkannte und in dem Umfange vertrat, wie sie erst seit noch gar nicht langer Zeit nunmehr von verschiedenen Seiten neuerlich propagiert wird.

Ein ausgezeichneter, sehr gewissenhafter Operateur, hatte Otto Küstner vor allem, wie schon angedeutet, an der Ausgestaltung der operativen Gynäkologie weitestgehenden Anteil. Allen den großen, um die Jahrhundertwende bereits eingeführten, aber noch vielfach zur Diskussion stehenden operativen Encheiresen unseres Fachs hat Otto Küstners Meisterhand ihren Stempel aufgedrückt, ihnen eigenes Ge-präge verliehen, so den Operationen wegen Lageveränderungen, Prolaps, Dammspalt, der Myomotomie, der Ovariotomie, der abdominalen Gebärmutterkrebsoperation und dem Kaiserschnitt. Bei letzterem führte Otto Küstner während der letzten 12-15 Jahre seiner Amtsperiode ein extraperitoneales Verfahren durch, das an seiner Klinik ausgezeichnete, bisher noch nicht übertroffene Resultate hinsichtlich der mütterlichen Lebenssicherheit zeitigte.

Ganz besonders Überragendes leistete seine Meisterhand auf plasti-schem Gebiete. Wir verdanken ihm die konservierende Operations-methode der Inversio uteri, und seine geniale Erkenntnis bescherte uns eine weitgehendst sicher zum Heilungserfolg führende Operations methode komplizierter Blasengenitalfisteln, die sogenannte metro-plastische Methode. Seine individualisierende Operationsmethode am Damm hat der exakten Muskel- und Fasziennaht des Beckenbodens und der isolierten Naht des Sphinkter ani beim totalen Dammspalt Eingang in die gynäkologische Akiurgie verschafft.

Otto Küstners ureigenstes Gebiet, worüber er ebenso wie über Pro-lapsoperationen auch ein Referat für die Deutsche Gesellschaft für Gynäkologie geliefert hat, war der Wundschut $\sum$ und alle die mit der Asepsis in Beziehung stehenden Fragen, wie die Gummihandschuh-prophylaxe, die Nahtmaterial-Sterilisierung, Operationssaalausrüstung und -ausgestaltung, Methodik der Schnittführung und Naht, intra-peritoneale Blutstillung, Drainage, Peritonisierung, Adhäsionsvermei-dung. An allem, was hier bleibendjgeschaffen wurde, hat Otto Küstner grundlegenden Anteil.

Durch die Einführung der von ihm als bakteriologische Sektion bezeichneten sterilen Sekretentnahme, unter Wahrung aller aseptischen Kautelen aus der Bauchhöhle soeben verstorbener Laparatomierter, konnte er zeigen, wie oft wir bei solchen unglücklich ausgegangenen Eingriffen einen Infektionsprozeß auch dann als Todesursache an-nehmen müssen, wo der Obduzent keine anatomischen Befunde für eine Infektion erheben kann. Ein so ausgezeichneter Operateur Otto Küstner war, so streng und eingehend war im Einzelfall seine Indikationsstellung, welche nicht nur die unmittelbaren Gefahren und den unmittelbaren Erfolg der Operation abwog, sondern auch die Frage späterer Folgen, Beschwerden, Nachschübe, Rezidive. So war er auch besonders zurückhaltend in der Indikationsstellung zu einem operativen Vorgehen bei entzündlichen Anhangsaffektionen; seine Anschauung, die sich so mancher moderne Operateur hier zum Wohle seiner Kranken zu eigen machen sollte, war: Hannes, $\mathrm{G}^{1} / 8 \mathrm{denkworte}$ an Otto Küstner. 421

Je später hier operiert wird, um so organerhaltender kann operiert werden und um so sicherer ist man vor Nachschüben bzw. Rezidiven.

Hatte aber Otto Küstner einen Eingriff für zweckdienlich erkannt, dann führte er ihn auch trotz widriger Verhältnisse durch. Hier möchte ich eines Verfahrens gedenken, das von ihm um die Jahrhundertwende angewandt wurde, dann aber bald in Vergessenheit geriet, welches m. E. aber jetzt gerade in der radiotherapeutischen Ära wieder in geeigneten Fallen angewandt werden sollte. Es ist die von ihm als Kolpokleisis rectalis bezeichnete Operation gewisser Fälle inoperabler Krebse. 
Ganz zu schweigen von noch vielen anderen seiner Leistungen und Werke, will ich auch hier nicht weiter eingehen auf Otto Küstners Verdienste um die Geburtshilfe, auf seine Lehre vom Dreiwehentypus bei der Steißlage, auf das obere Zervixsegment, auf die fötalen Miß-bildungen, die kindlichen intrapartalen Verletzungen, auf seine Stellung zur Nabelbehandlung und zur Behandlung der Nachgeburtsperiode, zur Episiotomie, auf Steißhaken und Rachiotom. Als Otto Küstner am 28. Februar 1923 vom Lehramt schied und sich nach Trossin zurückzog, schied er noch keineswegs von wissen-schaftlicher Forschung und Beschäftigung. Wenn wir ihn dann auch nur noch selten hier in unserem Kreis sahen und hörten, wie im Juni 1923 gelegentlich der Festsitzung zu Ehren seines ðOjährigen Doktorjubiläums, dann später zum 25jährigen Stiftungsfest unserer Gesellschaft und schließlich im Oktober 1929, gelegentlich der Feier seines 80. Geburtstags in unserer Gesellschaft, so hat sich Otto Küsíner, wie aus alien seinen Briefen an uns und aus gelegentlichen Unterhaltungen mit ihm an anderen Orten immer wieder deutlich hervorging, stets als zu uns hier nach Breslau gehörig gefühlt. Oft noch hat er in den letzten Jahren in mitteldeutschen gynäkologischen Gesellschaften und auf den Kongressen der Deutschen Gesellschaft für Gynäkologie das Wort ergriffen und bedeutsam die Diskussion durch seine Erfahrung und seine wissenschaftliche Ansicht beeinflußt. Gerade auch aus diesen Diskussionsreden leuchtet so voll und strahlend hell sein überragendes Gelehrtentum. Noch vor zwei Jahren erschienen Publikationen von ihm in unserer Fachpresse, und noch im Februar d. J. sprach er in der Leipziger Gesellschaft zur Diskussion.

Und in seinem Arbeitszimmer in Trossin arbeitete und schuf er unentwegt, ging er Problemen nach, die ihn zum Teil während seines ganzen arbeitsamen Lebens immer wieder beschäftigt und gefesselt hatten, so vor allem immer xmd immer wieder der Stieldrehung der Ovarientumoren, ihrer Genese und ihrer Gesetzmäßigkeit. Als dann ihn, den kòrperlichen und geistigen Hünen, schnell zunehmende, auf bulbären Verkalkungsprozessen beruhende, quälende Beschwerden Anfang April befielen, mußte er die ihm lieb und teuer gewesenen Arbeiten aus der Hand legen. Als dann am 12. Mai ihm der erlösende Tod die Feder für immer aus der Gelehrtenhand nahm, da fanden sich in seinem wissen-schaftlichen Nachlaß zwei nahezu abgesehlossene, umfängliche Werke über die Geschichte der Ovariotomie und über die von ihm ja auch besonders ausgebaute bimanuelle Untersuchung. Dieses wissenschaft-

422 Buchbesprechungen. - Personalien und Tagesnachrichten.

liche Vermächtnis wissen wir bei seinem Sohne Heinz Küsíner in besten Händen.

Jedes Wort der Trauer und Verehrung um Otto Küsíner ist zu wenig gesagt, jedes Wort der Dankbarkeit und der Würdigung dessen, was uns hier in der Gesellschaft und vor allem uns, seinen Schülern, Otto Küstner gab und war, ist ebenfalls zu wenig gesagt. Aber sein Name, sein Geist, seine Lehre wird uns weiter geleiten, soil und wird von uns weiter gefördert werden, von jedem nach seinen Kräften und an seinem Platz, solange selbst noch Atem in uns sein wird und sicherlich auch noch darüber hinaus in feme Zeiten. Um dies zu bekräftigen und dar-zutun, haben wir uns heute nicht an anderer Stelle, sondern ausdrüeklich hier in diesem Hörsaal, in welchem er fast 30 Jahre lehrte und wirkte, zu Otto Küstners Gedenken versammelt.

Buchbesprechungen.

Th. H. Tan de Velde: Der Ehespiegel. Ei $\prod$ Bilderbuch mit textlichen Erläute-rungen und Betrachtungen. Leipzig 1929. Grethlein u. Co.

Dieses „Bilderbuch” ist eine Sammlung von Bildern und Plastiken, welclie dem Verf. bei der Abfassung seiner Trilogie über die Ehe vor Augen gelegen haben. Die zu einem Ehespiegel zusammengefaßten Werke haben ihren Reflex in seinen Gedankeii gefunden. Sie sollen nicht als vollwertige Kunst und kulturhistorísche Studien aufgefaßt werden. Dieselben sollen mit den 
ihnen beigefügten Ausführungen Fachleute und auch Laien über gewisse für ihre Lebensgestaltung wichtige Punkte nachzudenken anregen. - Ref. kann nicht umhin, anzuerkennen, daß die Fülle dieser Bilder zu diesem Zweck vortrefflicb geeignet ist und zu Betrachtungen einladet.

A. Martin.

Personalien und Tagesnachrichten.

Privatdozent Dr. Hans Zacherl in Graz wurde als Nachfolger von Prof. Eymer zum o. Professor und Direktor der Universitäts-Frauen-klinik in Innsbruck ernannt.

Dr. S. Aschheim, Leiter des Laboratoriums der Universitäts-Frauen-klinik der Charité in Berlin, wurde zum Professor ernannt.

Prof. Dr. Graff-Panczowa in Wien hat einen Ruf als Ordinarius für Gynäkologie an die Universität in Iowa (Nordameríka) erhalten.

a. o. Prof. Dr. M. Seliga in Preßburg wurde zum ordentlichen Professor der Geburtshilfe und Gynäkologie ernannt.

Dr. Emit Heller in Luzern wurde an die neu zu errichtende geburts-hilflich-gynäkologische Abteilung am Kantonsspital berufen.

Wie uns soeben mitgeteilt wird, findet der III. Internationale Medizinische Fortbildungskurs der Tomarkin-Foundation New- York, Locarno, vom 11. bis 26. Oktober 1931 statt. Nähere Auskünfte erteilt bereitwilligst das Sekretariat der Tomarkin-Foundation, Locarno, Post-fach 128. 